\title{
Appeasing the Thirst for Rare-Earth Elements in Human Overpopulation with Novel Mining Strategies
}

\author{
Paris TE, Torres BE, Kim H, Janeway $\mathrm{K}$ and Heider D* \\ Department of Mathematics and Computer Science, Germany
}

ISSN: 2578-0255

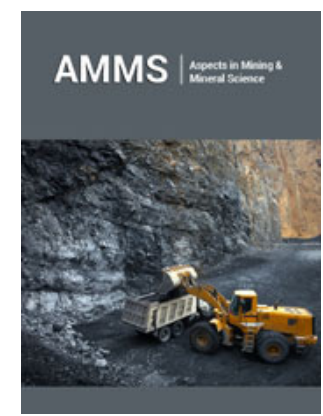

*Corresponding author: Dominik Heider, Department of Mathematics and Computer Science, Germany

Submission: 墭April 24, 2019

Published: 悳May 10, 2019

Volume 2 - Issue 5

How to cite this article: Paris TE, Torres BE, Kim H, Janeway K, Heider D. Appeasing the Thirst for Rare-Earth Elements in Human Overpopulation with Novel Mining Strategies. Aspects Min Miner Sci.2(5). AMMS.000549.2019.

DOI: 10.31031/AMMS.2019.02.000549.

Copyright@ Dominik Heider, This article is distributed under the terms of the Creative Commons Attribution 4.0 International License, which permits unrestricted use and redistribution provided that the original author and source are credited.

\section{Approach}

Human population has been rising continuously since the end of the Black Death, around the year 1350, although the most significant increase has been since the 1950s, mainly due to medical advancements and increases in agricultural productivity. While the rate of population growth has been declining since the 1980s, the absolute total numbers are still increasing. Recent rate increases in several countries previously enjoying steady declines are also apparently contributing to continued growth in total numbers. The United Nation (UN) population forecast of 2017 was predicting the total world population will be between 10-12 billion people by year 2100 .

Growing population comes with a higher need for goods, in particular improved wealth leads to higher demands in high end goods, such as smartphones or TVs, which require larger amounts of rare-earth elements. There are 17 rare-earth elements, namely cerium (Ce), dysprosium (Dy), erbium (Er), europium (Eu), gadolinium (Gd), holmium (Ho), lanthanum (La), lutetium ( $\mathrm{Lu})$, neodymium $(\mathrm{Nd})$, praseodymium $(\mathrm{Pr})$, promethium $(\mathrm{Pm})$, samarium $(\mathrm{Sm})$, scandium $(\mathrm{Sc})$, terbium $(\mathrm{Tb})$, thulium $(\mathrm{Tm})$, ytterbium $(\mathrm{Yb})$, and yttrium $(\mathrm{Y})$. Rareearth elements, except scandium, are heavier than iron and thus are produced by supernova nucleosynthesis or the s-process in asymptotic giant branch stars. In nature, spontaneous fission of uranium-238 produces trace amounts of radioactive promethium, but most promethium is synthetically produced in nuclear reactors. In 2011, Kato et al. [1] published results indicating the mud could hold rich concentrations of rare-earth minerals.

The uses, applications, and demand for rare-earth elements has expanded over the years. This is particularly due to the uses of rare-earth elements in low-carbon technologies. Some important uses of rare-earth elements, besides those mentioned before, are applicable to the production of high-performance magnets, catalysts, alloys, glasses, and electronics. Nd is important in magnet production. Rare-earth elements in this category are used in the electric motors of hybrid vehicles, wind turbines, hard disc drives, portable electronics, microphones, speakers. Ce and La are important as catalysts and are used for petroleum refining and as diesel additives. $\mathrm{Ce}, \mathrm{La}$, and $\mathrm{Nd}$ are important in alloy making, and in the production of fuel cells and Nickel-metal hydride batteries. $\mathrm{Ce}, \mathrm{Ga}$, and $\mathrm{Nd}$ are important in electronics and are used in the production of LCD and plasma screens, fiber optics, lasers, as well as in medical imaging. Additional uses for earth elements are as tracers in medical applications, fertilizers, and in water treatment. Unfortunately, mining, refining, and recycling of rare-earth elements have serious environmental consequences. A potential hazard could be low-level radioactive tailings resulting from the occurrence of thorium and uranium in rare-earth element ores Bourzac [2]. Improper handling of these substances can result in extensive environmental damage.

Thus, we propose mining of rare-earth elements on extra-terrestrial objects, e.g., asteroids or other planets. In particular planets with low-pressure atmosphere and lowmass with low gravitation, such as Mars (0.636 [0.4-0.87] $\mathrm{kPa}, 6.419 * 10^{3} \mathrm{~kg}$ ), are well suited. A permanent satellite with a high-energy light plow, e.g., phased array pulsed energy projectiles or a thalaron pulse, could be used to plough the surface. Due to the enormous heat (approximately 1273.15K) during explosion and the low-pressure atmosphere (Figure 1), the 
rare-earth elements are separated from the surrounding material and released into the atmosphere, eventually orbiting the object in highly enriched form and can easily collected by a low-energy repulsion beam [3-5]. The necessary energy for such a vehicle can be produced by several means, e.g., the use of Bussard reactor (BR) Bussard [6]. The BR uses an electromagnetic field to collect and compress hydrogen from the interstellar medium for a fusion reactor. Alternatives include the use matter-antimatter annihilation Jackson [7] with free deuterium and antideuterium Massam et al. [8] a singularity Hobson et al. [9], or Omega molecules [10-12], also named as particle 010, which can be synthesized with sufficient amounts of boronite ore. Using rare-earth element mining on extraterrestrial objects with the technological scheme developed in this study, opens the opportunity of virtually unlimited access without environmental damage, thus outperforming the current state-of-the-art mining of rare-earth elements. Moreover, our approach can be used also for mining of other materials having a melting point above $1273.15 \mathrm{~K}$, which is in fact true for most metals, such as gold (1336.15K) or platinum (2043.15K).

Figure 1: Scheme of the novel mining strategy.

A) probe orbiting the object in stratosphere.

B) detailed view of the probe.

\section{Funding}

This work was partially funded by Section 31, UFP, San Francisco, California.

\section{References}

1. Kato Y, Fujinaga K, Nakamura K, Takaya Y, Kitamura K, et al. (2011) Deep-sea mud in the Pacific Ocean as a potential resource for rare-earth elements. Nature Geoscience 4(8): 535-539.

1. Bourzac K (2010) Can the us rare-earth industry rebound? MIT Technology Review.

2. Modanese G (1996) Theoretical analysis of a reported weak gravitationalshielding effect. EPL (Europhysics Letters) 35(6): 413.

3. Ning W (2006) Mechanism of gravity impulse. Communications in theoretical Physics 46(4): 639.

4. Chen J, Ng J, Lin Z, Chan CT (2011) Backward pulling force from a forward propagating beam. DOI: 10.1038/nphoton.2011.153.

5. Bussard RW (1960) Galactic matter and interstellar sight. Astronautica Acta 6(4): 179-194.
6. Jackson A (1980) Some considerations on the antimatter and fusion ram augmented interstellar rocket. Journal of the British Interplanetary Society 33: 117-120.

7. Massam T, Muller T, Righini B, Schneegans M, Zichichi A (1965) Experimental observation of antideuteron production. Il Nuovo Cimento A 63(1): 10-14.

8. Hobson MP, Efstathiou GP, Lasenby AN (2006) General relativity: An introduction for physicists. Cambridge University Press, UK.

9. Aaij R, Adeva B, Adinolfi M, Ajaltouni Z, Akar S, et al. (2017) Observation of five new narrow $\mathrm{w}^{0}{ }_{\mathrm{c}}$ states decaying to $\mathrm{E}_{\mathrm{c}}^{+} \mathrm{k}_{-}$. Physical review letters 118(18): 182001.

10. Abazov VM, Abbott B, Abolins M, Acharya BS, Adams M, et al. (2008) Observation of the doubly strange b baryon Omegab. Phys Rev Lett 101(23): 232002.

11. Ketteract J (1998) Chemical synthesis of highly energetic omega molecules from Boronite ore. In Proceedings of $23^{\text {rd }}$ International Energy Conference Lantaru. 